San Jose State University

SJSU ScholarWorks

Master's Theses

Master's Theses and Graduate Research

1994

\title{
The perception of ventilatory threshold and its accuracy for indicating anaerobic threshold
}

Howard Bennett Lieberman

San Jose State University

Follow this and additional works at: https://scholarworks.sjsu.edu/etd_theses

\section{Recommended Citation}

Lieberman, Howard Bennett, "The perception of ventilatory threshold and its accuracy for indicating anaerobic threshold" (1994). Master's Theses. 928.

DOI: https://doi.org/10.31979/etd.vc3f-n7p5

https://scholarworks.sjsu.edu/etd_theses/928

This Thesis is brought to you for free and open access by the Master's Theses and Graduate Research at SJSU ScholarWorks. It has been accepted for inclusion in Master's Theses by an authorized administrator of SJSU ScholarWorks. For more information, please contact scholarworks@sjsu.edu. 


\section{INFORMATION TO USERS}

This manuscript has been reproduced from the microfilm master. UMI films the text directly from the original or copy submitted. Thus, some thesis and dissertation copies are in typewriter face, while others may be from any type of computer printer.

The quality of this reproduction is dependent upon the quality of the copy submitted. Broken or indistinct print, colored or poor quality illustrations and photographs, print bleedthrough, substandard margins, and improper alignment can adversely affect reproduction.

In the unlikely event that the author did not send UMI a complete manuscript and there are missing pages, these will be noted. Also, if unauthorized copyright material had to be removed, a note will indicate the deletion.

Oversize materials (e.g., maps, drawings, charts) are reproduced by sectioning the original, beginning at the upper left-hand corner and contimuing from left to right in equal sections with small overlaps. Each original is also photographed in one exposure and is included in reduced form at the back of the book.

Photographs inchuded in the original manuscript have been reproduced xerographically in this copy. Higher quality $6^{n} \times 9^{n}$ black and white photographic prints are available for any photographs or illustrations appearing in this copy for an additional charge. Contact UMI directly to order.

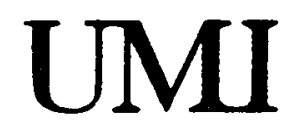

A. Bell \& Howell intormation Company 300 North Zeeb Road. Ann Afbor. MI 48106-1346 USA

$313: 761-4700 \quad 800: 521-0600$ 


\title{
THE PERCEPTION OF VENTILATORY THRESHOLD AND ITS ACCURACY FOR INDICATING ANAEROBIC THRESHOLD
}

\author{
A Thesis \\ Presented to \\ The Faculty of the Department of Human Performance \\ San Jose State University
}

\author{
In Partial Fulfillment \\ of the Requirements for the Degree \\ Master of Arts
}

By

Howard Bennett Lieberman

December, 1994 
UMI Number : 1361186

\section{Copyright 1994 by Lieberman, Howard Bennett All rights reserved.}

UWI Microform Edition 1361186

Copyright 1995, by UMI Company. All rights reserved.

This microform edition is protected against unauthorized copying under Title 17 , United states Code.

\section{UMI}

300 North Zeeb Road

Ann Arbor, MI 48103 
c 1994

Howard Bennett Lieberman

ALL RIGHTS RESERVED 
APPROVED FOR THE DEPARTMENT OF HUMAN PERFORMANCE

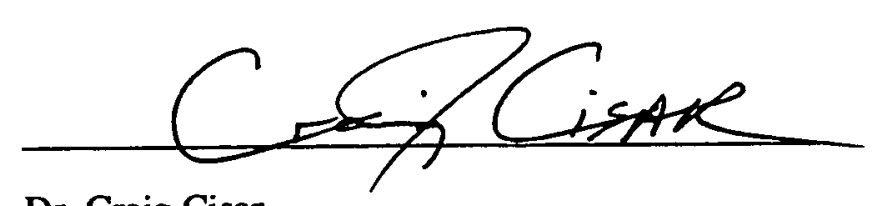

Dr. Craig Cisar

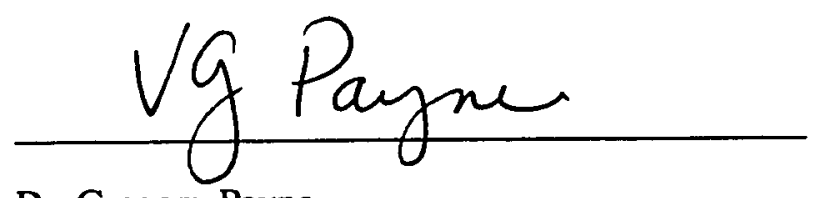

Dr. Gregory Payne

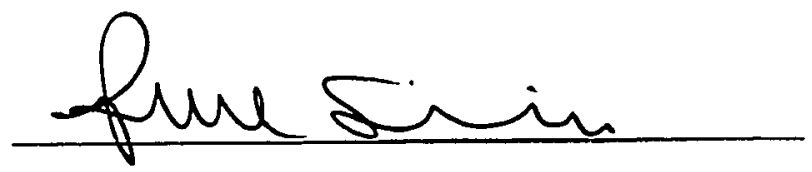

Dr. Phil Sienna

APPROVED FOR THE UNIVERSITY

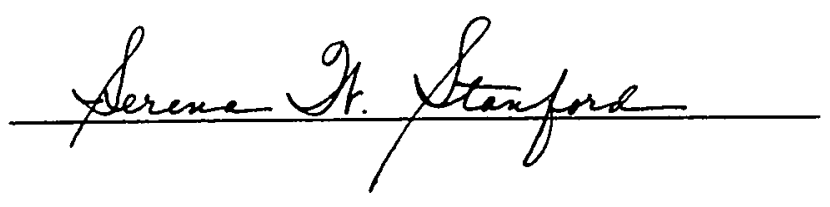




\section{ABSTRACT \\ THE PERCEPTION OF VENTILATORY THRESHOLD AND ITS ACCURACY FOR INDICATING ANAEROBIC THRESHOLD}

by Howard Bennett Lieberman

The purpose of this study was to determine if exercising subjects could accurately detect abrupt changes in ventilation associated with the ventilatory threshold (VT) and lactate threshold (LT). Twelve trained males, 24 to 36 years old, performed incremental cycle ergometry to voluntary exhaustion. Expired ventilation rate (VE), ventilatory frequency (VF), tidal volume (TV), heart rate (HR), oxygen uptake rate (VO2), and blood lactate concentration (LA) were measured each minute. Subjects identified perceived ventilatory threshold (PVT) when they noticed abrupt changes in their ventilation beyond those occuring with increasing workloads. VT and LT were determined. Strong correlations were found between VO2 at PVT and VT ( $r=.85)$, PVT and LT ( $r=.88)$, and VT and LT ( $(\underline{r}=.92)$. VE at PVT was related to VF at PVT $(\underline{I}=.79)$ and TV at PVT $(\underline{r}=.68)$. One-way analyses of variance and Tukey post-hoc analyses revealed that VE, VO2, HR, and TV at PVT were lower $(\underline{p}<.05)$ than at VT. No significant $(\underline{p}<.05)$ differences were observed in the physiological variables measured between PVT and LT or VT and LT. PVT was highly related to VT and LT, but more strongly related to LT. PVT appears to be a useful indicator of anaerobic threshold. 


\section{Acknowledgements}

I would like to thank the following people for their assistance during this entire project: Craig Cisar, commitee chair, from whose guidance I have benefited since my first graduate course at SJSU; Joanna Losito, without whom the most critical laboratory measurements in this study could not have been performed; Mike Cole, who volunteered an extra set of skilled hands for data collection; all of my subjects; my brothers and parents; and my wife Mindy for her persistent encouragement and unconditional support throughout my graduate school career. 
Table of Contents

Abstract

page

Acknowledgements

iv

List of Tables

viii

Chapter 1: Introduction

1

Background for the Study 1

Statement of the Problem 3

Statement of Purpose

Statement of the Hypotheses 4

Delimitations 5

Limitations $\quad 5$

Assumptions $\quad 6$

Definitions 6

$\begin{array}{ll}\text { Chapter 2: Review of Literature } & 9\end{array}$

The Gas Exchange Anaerobic Threshold 9

Challenging the Gas Exchange Model 12

$\begin{array}{ll}\text { Summary } & 16\end{array}$

$\begin{array}{ll}\text { Chapter 3: Methods } & 17\end{array}$

$\begin{array}{ll}\text { Subjects } & 17\end{array}$

$\begin{array}{ll}\text { Instrumentation } & 17\end{array}$

$\begin{array}{ll}\text { Procedures } & 18\end{array}$

$\begin{array}{ll}\text { Measurements } & 19\end{array}$

$\begin{array}{ll}\text { Statistical Analysis } & 21\end{array}$ 
Chapter 4: Results

Analysis of Data $\quad 22$

Summary of Findings $\quad 29$

Discussion of Findings $\quad 30$

$\begin{array}{ll}\text { Conclusions } & 33\end{array}$

$\begin{array}{ll}\text { Practical Applications } & 33\end{array}$

Weaknesses of the Study 33

Recommendations for Future Research 34

$\begin{array}{ll}\text { References } & 35\end{array}$

$\begin{array}{lr}\text { Appendices } & 39\end{array}$ 


\section{List of Tables}

page

Table 1 Descriptive Characteristics of the Subjects 23

Table 2 Zero Order Correlation Matrix for VO2 at PVT, VT, and LT 24

Table 3 Zero Order Correlation Matrix for VE at PVT, VT, and LT 24

Table 4 Zero Order Correlation Matrix for VF at PVT, VT, and LT 25

Table 5 Zero Order Correlation Matrix for TV at PVT, VT, and LT 25

Table 6 Zero Order Correlation Matrix for Time at PVT, VT, and LT 26

Table 7 Correlation Matrix for VF and VE at PVT, VT, and LT 26

Table 8 Correlation Matrix for TV and VE at PVT, VT, and LT 27

Table 9 Results of Repeated Measures ANOVA and Tukey post hoc analyses 


\section{Chapter I}

\section{Introduction}

This chapter is divided into the following sections: background for the study, statement of the problem, statement of purpose, hypotheses, delimitations, limitations, assumptions, and definitions.

\section{Background for the Study}

The Physiological Models. Rates of ventilation and circulation are functionally linked to metabolic demands of cells. During increasing exercise from rest up to between $40 \%$ and $60 \%$ of maximum aerobic capacity, ventilation increases linearly with increasing oxygen consumption (Davis, 1985; McArdle, Katch, \& Katch, 1991; Skinner \& McLellan, 1980; Wasserman, 1978). However, above this point, ventilation increases nonlinearly in relation to metabolic demand. This abrupt upward swing has been termed the gas exchange or ventilatory anaerobic threshold. This represents the respiratory compensation to events directly linked to increased anaerobic glycolytic metabolism and the accumulation of lactate in the blood (Davis, 1985; McArdle et al., 1991; Skinner \& McLellan, 1980; Wasserman, 1978).

During exercise, lactate produced in working skeletal muscle diffuses into the blood. From rest up through moderate exercise intensities corresponding to $40 \%$ of maximum aerobic power, lactate concentration remains slightly above resting levels. At these work rates lactate produced metabolically is matched by clearance in the liver and by oxidation to pyruvate in muscle for entry into the Kreb's cycle (McArdle et al., 1991; Skinner \& McLellan, 1980).

For most individuals, as exercise intensity increases to between $40 \%$ and $60 \%$ of maximum, lactate concentration systematically rises. This abrupt upswing in blood lactate 
concentration above resting levels has been termed the lactate anaerobic threshold (Brooks, 1985; Davis et al., 1983; Karlsson \& Jacobs, 1982). At this time, the plasma sodium bicarbonate ( $\mathrm{NaHCO} 3)$ buffering system becomes the primary mechanism for the maintenance of blood pH (Davis, 1985; McArdle et al., 1991, Skinner \& McLellan, 1980). Once in the blood, metabolic lactate $(\mathrm{HLa})$ rapidly disassociates and reacts with sodium bicarbonate to form sodium lactate (NaLa) and carbonic acid (H2CO3). Carbonic acid rapidly disassociates into an acid $(\mathrm{H}+)$ and bicarbonate ion (HCO3-). In the presence of the enzyme carbonic anhydrase, $\mathrm{H} 2 \mathrm{CO} 3$ - disassociates and forms water $(\mathrm{H} 2 \mathrm{O})$ and carbon dioxide (CO2) (Davis, 1985; McArdle et al., 1991; Skinner \& McLellan, 1980).

The resulting stimulus for increased ventilation is twofold. Increased $\mathrm{CO} 2$ concentration in the blood from the buffering reactions adds to metabolically produced $\mathrm{CO} 2$. Ventilatory drive is increased to remove the excess $\mathrm{CO} 2$. Increased circulating free $\mathrm{H}+$ ions from the buffering reactions stimulate the carotid bodies to increase ventilation and prevent a fall in blood pH (Davis, 1985; McArdle et al., 1991). Because of their sensitivity to $\mathrm{H}+$ ions, these peripheral chemoreceptors provide the dominant control of ventilation during moderate and heavy exercise (Wasserman, 1978; Whipp, 1983; Whipp \& Davis, 1979). The importance of this mechanism has been confirmed in studies where patients' carotid bodies were removed and the resultant ventilatory responses to heavy exercise were greatly reduced (Wasserman et al., 1975).

In summary, the buffering mechanism increases $\mathrm{CO} 2$ and $\mathrm{H}+$ concentration in the blood beyond that produced metabolically. This stimulates a disproportionate increase in ventilation above that created by the metabolic demand of muscular work.

However, the gas exchange model of anaerobic threshold has been challenged as being an overly simplistic explanation of two indirectly related phenomena (Brooks, 1985). Ventilatory threshold may be the result of several complex and linked causes. Expired 
ventilation rate may not track changes in blood lactate concentration and any observed correlations may be coincidental (Brooks, 1985).

Researchers have also suggested that since oxygen is lentiful at submaximal workloads, the lactate threshold may only reflect a change in the balance between glycolytic and oxidative metabolism (Hagberg, 1984; Skinner \& McLellan, 1980). This may be related to the capacity of respiratory enzymes in the muscle mitochondria rather than an indication of a complete shift to anaerobic metabolism resulting from cellular hypoxia. Additionally, Skinner and McLellan (1980) proposed this shift was primarily the result of changes in muscle fiber type recruitment as exercise intensity increased.

Monitoring Exercise Intensity. Heart rate monitoring has been the traditional benchmark for determining relative exercise intensity (McArdle et al., 1991). However, perceptual cues from both ventilatory volume and frequency have also been shown to be important indicators of exercise intensity (Mihevic, 1981). These parameters are especially valuable sources of sensory information relating to physical effort during high and peak exercise intensities (Mihevic, 1981). Specifically, tachypnea has been identified as a key feature of the respiratory compensation to metabolic acidosis at high levels of exercise (Wasserman, 1978).

An individual's aerobic fitness level determines the relative exercise intensity and exercise heart rate at which metabolic acidosis occurs. A person with high aerobic capacity can exercise at a greater percentage of their maximum aerobic power than a less fit person before the accumulation of blood lactate. Therefore, the lactate threshold can be an important indicator of endurance capacity (Hagberg, 1984; McArdle et al., 1991). Statement of the Problem

As an individual's aerobic capacity improves or declines, the percentage of maximum aerobic power that elicits lactate threshold will change. Periodic physiological 
exercise testing that directly measures blood lactate concentrations is necessary to adjust the training level that represents the lactate threshold. However, if ventilatory threshold is the respiratory compensation to the accumulation of blood lactate, then its occurrence will remain linked to the lactate threshold regardless of fluctuations in aerobic capacity. If exercising subjects can reliably perceive the change in ventilation associated with the ventilatory threshold, then a dependable mechanism for high intensity exercise monitoring could be used throughout an individual's changing level of aerobic fitness. This ability would allow accurate monitoring of high exercise intensities during aerobic endurance training without direct measurement of heart rate or blood lactate. This technique would be especially valuable for both recreational and competitive athletes who are unable to undergo regular physiological and biochemical testing.

\section{Statement of Purpose}

The purpose of this study was to determine whether or not exercising subjects could accurately detect the disproportionate increase in ventilation associated with the ventilatory threshold. This study also examined whether the perception of ventilatory threshold could be used as an accurate indicator of the lactate threshold. Additionally, the relationship between ventilatory and lactate thresholds was reassessed.

\section{Statement of the Hypotheses}

The following hypotheses were tested in this study:

1. The oxygen uptake rate (VO2), heart rate (HR), expired ventilation rate (VE), ventilatory frequency (VF), tidal volume (TV), blood lactate concentration (LA), rating of perceived exertion (RPE), percent of maximal oxygen uptake rate (\%VO2 max), and percent of maximal heart rate (\%HR max) at the perception of ventilatory threshold are not significantly different from the VO2, HR, VE, VF, TV, LA, RPE, \%VO2 max, and \%HR max at true gas exchange ventilatory threshold. 
2. The VO2, HR, VE, VF, TV, LA, RPE, \%VO2 max, and \%HR max at the perception of ventilatory threshold are not significantly different from the VO2, HR, VE, VF, TV, LA, RPE, \%VO2 max, and \%HR max at lactate threshold.

3. The VO2, HR, VE, VF, TV, LA, RPE, \%VO2 max, and \%HR max at true gas exchange ventilatory threshold are not significantly different than the VO2, HR, VE, VF, TV, LA, RPE, \%VO2 max, and \%HR max at lactate threshold.

4. The VO2, HR, VE, VF, TV, and time at the perception of ventilatory threshold are not strongly related to the VO2, HR, VE, VF, TV, and time at true gas exchange ventilatory threshold.

5. The VO2, HR, VE, VF, TV, and time at the perception of ventilatory threshold are not strongly related to the VO2, HR, VE, VF, TV, and time at lactate threshold.

6. The VO2, HR, VE, VF, TV, and time at true gas exchange ventilatory threshold are not strongly related to the VO2, HR, VE, VF, TV, and time at lactate threshold.

7. The ventilatory frequencies at the perception of ventilatory threshold, true gas exchange ventilatory threshold, or lactate threshold are not strongly related to the expired ventilation rates at the perception of ventilatory threshold, true gas exchange ventilatory threshold, or lactate threshold.

8. The tidal volumes at the perception of ventilatory threshold, true gas exchange ventilatory threshold, or lactate threshold are not strongly related to the expired ventilation rates at the perception of ventilatory threshold, true gas exchange ventilatory threshold, or lactate threshold.

Delimitations

This study was delimited to healthy, aerobically trained males aged 18 to 40 years. Limitations

Factors not controlled for and limiting the interpretation of results from this study 
included the subjects' motivation, nutritional status, level of fatigue, and genetic make-up. Assumptions

All subjects were assumed to perform their best effort during testing and to have understood all pre-test instructions.

\section{Definitions}

The following definitions are defined for the purpose of this study:

Anaerobic Threshold (AT). The time or point during the progressive exercise test at which blood lactate accumulates non-linearly and its concentration in the blood increases steadily from resting levels. Addtionally, anaerobic threshold has been described as the point during progressive exercise at which a non-linear increase in expired ventilation rate (VE) occurs. AT has been used to describe both the individual and associated occurrence of the lactate and ventilatory thresholds described below (Brooks \& Fahey, 1984; McArdle et al., 1991).

Carbon dioxide production rate (VCO2). The volume of carbon dioxide expired each minute expressed in liters per minute (McArdle et al., 1991).

Expired ventilation rate (VE). The volume of air expired each minute expressed in liters per minute (McArdle et al., 1991).

Fraction of expired carbon dioxide (FeCO2). Fraction of carbon dioxide in expired air expressed as a percentage of the total expired volume (Skinner \& McLellan, 1980).

Fraction of expired oxygen (FeO2). Fraction of oxygen in expired air expressed as a percentage of the total expired volume (Skinner \& McLellan, 1980).

Lactate threshold (LT). The time or point during the progressive exercise test at which blood lactate accumulates non-linearly and its concentration in the blood increases steadily from resting levels (Brooks, 1985; Davis et al., 1983; Karlsson \& Jacobs, 1982; McArdle et al., 1991). 
Maximal oxygen uptake rate (VO2 max). The time or point during the progressive exercise test where a plateau or decrease in oxygen uptake rate (VO2) occurs with increasing workloads. Additionally, exercising subjects will be within ten beats per minute of their age-predicted maximum heart rate and/or will have a respiratory exchange ratio ( $R$; VCO2/VO2) equal to or greater than 1.00 (American College of Sports Medicine [ACSM], 1991; McArdle et al., 1991).

Metabolic Acidosis. An increase in $\mathrm{H}+$ concentration and decrease in muscle and blood $\mathrm{pH}$ during progressive exercise resulting from the accumulation of blood lactate (McArdle et al., 1991).

Oxygen uptake rate (VO2). The volume of oxygen consumed each minute expressed in liters per minute or milliliters per kilogram per minute (McArdle et al., 1991).

Perceived ventilatory threshold (PVT). The time or point during the progressive exercise test at which the exercising subject perceives the disproportionate increase in expired ventilation rate described below as the ventilatory threshold. The time for PVT was determined to be the minute within which detection of ventilatory changes were signaled by the subject; that is, a signal at time eleven minutes, fourteen seconds would be recorded as PVT occurring in the eleventh minute. A signal at time eleven minutes, forty-seven seconds would also be recorded as PVT occurring in the eleventh minute.

Respiratory exchange ratio ( $\mathrm{R} ; \mathrm{VCO} / \mathrm{VO} 2)$. The ratio of the volume of carbon dioxide expired per minute to the volume of oxygen consumed per minute (McArdle et al., 1991).

Tidal Volume (TV). The volume of air inspired or expired each breath expressed as liters per breath (McArdle et al., 1991).

Ventilatory equivalent for carbon dioxide (VE/VCO2). The ratio of expired ventilation rate to carbon dioxide production rate (McArdle et al., 1991). 
Ventilatory equivalent for oxygen (VE/VO2). The ratio of expired ventilation rate to oxygen uptake rate (McArdle et al., 1991).

Ventilatory frequency (VF). Breathing frequency expressed as breaths per minute (McArdle et al., 1991).

Ventilatory threshold (VT). The time or point during the progressive exercise test where the following criteria occur: 1) a systematic increase in the mathematically determined standard error of estimate between VE and VO2; 2) a nonlinear increase in expired ventilation rate (VE); 3 ) the fraction of expired $\mathrm{O} 2(\mathrm{FeO} 2)$ reaches its minimum and the fraction of expired $\mathrm{CO} 2$ (FeCO2) reaches its maximum; 4) a nonlinear increase in carbon dioxide production rate ( $\mathrm{VCO} 2)$; and 5) a systematic increase in the ventilatory equivalent for oxygen (VE/VO2) without a change in the ventilatory equivalent for carbon dioxide (VE/VCO2) (Caiozzo et al., 1982; James, Adams, \& Wilson, 1989; Powers, Dodd, \& Garner, 1984; Skinner \& McLellan, 1980; Wasserman et al., 1973). Criteria four and five were only used when criteria one, two, and three were inconclusive. 


\section{Chapter II \\ Review of Literature}

Introduction

In this chapter, the literature that has evaluated the relationship between the ventilatory and lactate thresholds is reviewed. Controversy exists regarding the relationship between the accumulation of blood lactate and the nonlinear changes in ventilatory parameters that occur during progressive exercise.

The Gas Exchange Anaerobic Threshold

The classical view of anaerobic threshold proposed that changes in certain gas exchange parameters occurring during exercise are directly related to the changes in blood $\mathrm{pH}$ associated with the accumulation of blood lactate (Wasserman, Whipp, Koyal, \& Beaver, 1973). This suggested that noninvasive measurement of these parameters could serve as a means of detecting the onset of metabolic acidosis.

Almost twenty years ago, the alterations in gas exchange during exercise were evaluated as a noninvasive means of detecting metabolic acidosis by Wasserman et al., (1973). Eighty-five healthy male and female subjects, aged 17 to 91 , performed incremental exercise to maximum workloads on a cycle ergometer. Gas analysis continuously measured concentrations of expired $\mathrm{O} 2$ and $\mathrm{CO} 2$. Expired ventilation rate (VE), oxygen uptake rate (VO2), carbon dioxide production rate (VCO2), fraction of $\mathrm{O} 2$ in expired gases $(\mathrm{FeO} 2)$ and fraction of $\mathrm{CO} 2$ in expired gases ( $\mathrm{FeCO} 2)$, and the respiratory exchange ratio (R; VCO2/VO2) were calculated. Anaerobic threshold (AT) was identified as the point during exercise at which VE increased nonlinearly, VCO2 increased nonlinearly, and $\mathrm{FeO} 2$ increased without a concurrent decrease in $\mathrm{FeCO} 2$.

VE was shown to be the most sensitive and $R$ the least sensitive predictor of AT. 
The researchers determined VE to be the most sensitive in responding to $\mathrm{pH}$ changes in the blood and the increase in arterial $\mathrm{PCO} 2$ from the disassociation of bicarbonate ion during lactate buffering. However, blood samples were not measured directly so direct comparisons could not be made between expired ventilation rate, blood lactate concentrations, blood gases, and blood pH changes.

Ventilatory responses to metabolic acidosis have also been examined and compared for cycle ergometry and treadmill running (Koyal, Whipp, Huntsman, Brey, \& Wasserman, 1976). Twenty healthy male and female subjects, 20 to 33 years old, performed cycle ergometry at three different workloads. Subjects then performed treadmill running at three equivalent workloads. During the last minute of each 15 minute work bout, VE, VO2, and VCO2 were measured. Blood samples were also drawn at the same time and analyzed for $\mathrm{pH}, \mathrm{CO} 2$ concentration (PCO2), $\mathrm{O} 2$ concentration (PO2), lactate (LA) concentration, and plasma bicarbonate ion ( $\mathrm{HCO}-)$ concentration.

Results showed that at equal VO2's a higher VE and LA concentration but a lower HCO3- concentration occurred during cycle ergometry. Additionally, the increase in VE was a mirror image of the decrease in $\mathrm{HCO} 3$ - during both cycle ergometry and treadmill running.

The researchers concluded that the increase in VE represented respiratory compensation to metabolic acidosis. This conclusion was reinforced by the simultaneous and linearly related decline in plasma bicarbonate buffer and increase in blood lactate concentration.

To determine the most sensitive indicator of anaerobic threshold, a comparison of several gas exchange indices used to predict anaerobic threshold was performed (Caiozzo et al., 1982). Sixteen 20 to 31 year old male and female subjects were studied. During two separate maximal cycle ergometer tests for each subject, VE, VCO2, R, and the ventilatory 
equivalents for oxygen and carbon dioxide (VE/VO2 and VE/VCO2) were measured every 30 seconds. Blood lactate concentrations were also measured every 30 seconds. The systematic increase in lactate concentration above resting levels (lactate threshold) was used as the criterion measure for anaerobic threshold. The gas exchange (ventilatory) anaerobic threshold was defined as the point where there was a nonlinear increase in VE, VCO2, R, and VE/VO2 without an increase in VE/VCO2.

The highest correlation between any gas exchange measure for anaerobic threshold and the lactate threshold was found for VE/VO2 ( $I=.93, \mathrm{p}<.001)$. The lowest correlation was for $\mathrm{R}$ and the lactate threshold $(\mathrm{r}=.39, \mathrm{p}<.05)$. Additionally, $\mathrm{VE}$ and $\mathrm{VCO} 2$ threshold measures correlated highly ( $r=.88$ and .83 , respectively) with lactate threshold measures $(\mathbf{p}<.001)$. For test-retest reliability, VE/VO2 was also the best indicator of lactate anaerobic threshold $(\mathrm{r}=.93, \mathrm{p}<.001)$. The researchers concluded that VE/VO2 was the most accurate and reliable noninvasive index of anaerobic threshold.

In 1984, Powers, Dodd, and Garner also measured the precision with which gas exchange variables could predict anaerobic threshold using lactate threshold as the criterion measure. Thirteen trained male subjects with a mean $\mathrm{VO} 2 \max$ of $45 \mathrm{ml} / \mathrm{kg} / \mathrm{min}$ performed incremental cycle ergometry. To allow for complete lactate diffusion from working muscle into the bloodstream, workloads were increased every three minutes. Breath by breath gas analysis continuously measured $\mathrm{VE}, \mathrm{FeO} 2$, and $\mathrm{FeCO}$. VO2, VCO2, VE/VO2, VE/VCO2, and heart rate were calculated for each workload. Blood lactate concentrations were also measured during the last 30 seconds of each workload. Lactate anaerobic threshold $(\mathrm{LT})$ was defined as the systematic increase in blood lactate concentration with continued exercise. Ventilatory anaerobic threshold (VT) was defined as the nonlinear increase in VE and VE/VO2 in relation to VO2 without a concurrent rise in VE/VCO2. Ventilatory and lactate anaerobic thresholds were correlated. 
Results showed moderate correlations between lactate threshold and both ventilatory measures $(\underline{\underline{r}}=.67, \underline{p}<.05$ for VE and $\mathrm{LT} ; \underline{\underline{r}}=.63, \underline{\mathrm{p}}<.05$ for VE/VO2 and LT). Lactate and ventilatory thresholds occurred separately in five of the thirteen subjects. However, in nine of the subjects, the lactate and VE thresholds occurred at exactly the same VO2. In eight of the subjects lactate and VE/VO2 thresholds occurred together. In six of the subjects all three parameters occurred at exactly the same VO2. Though gas exchange measures did not accurately determine lactate threshold in all subjects, it was a precise measure of lactate threshold in some individuals.

More recently, the relationship between gas exchange anaerobic threshold and ventilatory frequency was examined (James, Adams, \& Wilson, 1989). Thirteen healthy active males and females, aged 21 to 44 years, performed incremental cycle ergometry. Breath by breath expired $\mathrm{FeO} 2, \mathrm{FeCO} 2, \mathrm{VE}, \mathrm{VO} 2$, and $\mathrm{VCO} 2$ were measured as well as ventilatory frequency (VF) and heart rate during each minute of the test. The anaerobic threshold criterion was defined as the VO2 at which a nonlinear increase in VE with respect to VO2 occurred. The nonlinear rise in VF with respect to VO2 was compared and correlated to this criterion.

A correlation of $\underline{\underline{x}}=.83(\underline{p}<.05)$ was found between the VE threshold and VF threshold. The researchers concluded that the disproportionate change in ventilatory frequency could be used as an accurate indicator of the ventilatory anaerobic threshold and proposed its use as a means of monitoring exercise intensity. However, in this study the exercising subjects were not asked to detect this change and blood lactate concentrations were not measured.

\section{Challenging the Gas Exchange Model}

The link between ventilatory and lactate thresholds may be coincidental. The theory of a respiratory hyperventilation response to metabolic acidosis was challenged in a study 
that compared ventilatory and lactate responses between normal subjects and patients having McArdle's disease (Hagberg et al., 1982). McArdle's disease is a rare genetic disorder where patients lack the muscle enzyme phosphorylase and are unable to increase lactate and thus hydrogen ions $(\mathrm{H}+)$ during exercise.

Four patients and twenty six normal subjects performed incremental cycle ergometry to maximum voluntary exhaustion. $\mathrm{VO} 2, \mathrm{VCO} 2, \mathrm{VE}, \mathrm{R}, \mathrm{FeCO} 2$, and $\mathrm{FeO} 2$ were monitored breath by breath throughout the testing. Venous blood samples were drawn during each minute of exercise and analyzed for lactate concentration and $\mathrm{pH}$.

Test results indicated that the expected accumulation of blood lactate in the normal subjects began at approximately $65-75 \%$ of VO2 max. Also as expected, the McArdle's patients showed no lactate response above resting levels during their exercise tests. Accordingly, blood pH for normal subjects decreased significantly after lactate concentration increased. Blood $\mathrm{pH}$ for the McArdle's patients remained unchanged.

The critical finding in this study concerned the similar ventilatory responses in both groups of subjects. Both groups demonstrated a nonlinear and abrupt increase in ventilation at a similar percentage of their VO2 max. Interestingly, hyperventilation in the McArdle's disease patients was associated with an increase rather than a decrease in blood $\mathrm{pH}$.

These findings contradicted the premise that ventilatory threshold is the result of metabolic acidosis occuring with the accumulation of blood lactate. The researchers suggested that because of the complete disassociation between lactate and ventilatory thresholds in the McArdle's patients, hyperventilation with intensive exercise is primarily stimulated by factors other than increased $\mathrm{H}+$ concentration and lowered blood $\mathrm{pH}$.

Continuous and interval training have also been shown to elicit different responses in the lactate and ventilatory thresholds during incremental exercise (Poole \& Gaesser, 
1985). Seventeen sedentary young men participated in an eight week, three times per week training program following one of three training protocols. Subjects either exercised continuously for 55 minutes at $50 \%$ of VO2 max (group 1), continuously for 35 minutes at $70 \%$ of VO2 max (group 2), or performed ten two minute intervals at $105 \%$ of VO2 max with each interval separated by a two minute recovery period (group 3). Subjects were tested before training, after four weeks, and after eight weeks of training. During each test, ventilatory and gas exchange measures were recorded breath by breath using an integrated computer and gas analysis system. VE, VO2, VCO2, VE/VO2, VE/VCO2, R, FeO2, and FeCO2 were calculated. Blood lactate was measured each minute of the test. Ventilatory threshold (VT) was defined as the VO2 associated with the workload where VE/VO2 began to systematically rise without a concurrent rise in VE/VCO2. Lactate threshold (LT) was defined as the VO2 associated with the workload at which blood lactate began to systematically rise above resting levels.

Training increased VO2 max, VT, and LT in all groups. However, the magnitude of the increase of VT in group 3 (interval training) was significantly greater than groups 1 and 2. Interestingly, the change in LT was similar in all groups. However, in group 1 the LT change was 2.5 times greater than the corresponding change in VT. This study showed different LT and VT responses to continuous and interval training. The authors suggested that alterations in VT and LT were regulated by different mechanisms and that lactic acidosis may have only been one of many factors influencing ventilation.

In a follow-up to the study discussed above, Gaesser and Poole (1986) supported their contention that different mechanisms underlie the regulation of LT and VT. They examined the different time courses of adaptation for the ventilatory and lactate thresholds in six healthy untrained subjects. For three weeks, six days per week, thirty minutes per day, subjects exercised at $70-80 \%$ of a pretraining test VO2 max. At the end of each week, 
subjects performed an incremental cycle ergometer test. During each test ventilatory gas exchange parameters were measured as described in the above studies. Blood samples were analyzed for lactate. Both ventilatory and lactate thresholds were determined by the previously mentioned criteria.

After three weeks of training, significant increases in VO2 max (11.1\%) and lactate threshold (29.3\%) were recorded. However, ventilatory threshold did not increase significantly even though pretraining tests showed that the VO2 at VT and LT were not significantly different. The results of this study showed that significant changes in lactate threshold can occur without significant changes in ventilatory threshold. This data supported results from Poole and Gaesser's (1985) previous research which showed that VT and LT respond differently to different types of training protocols.

Caffeine has also been shown to alter the relationship between LT and VT (Berry, Stoneman, Weyrich, \& Burney, 1991). Ten healthy, young adult male subjects performed two incremental treadmill tests. Forty-five minutes prior to testing subjects consumed a capsule containing either the equivalent of seven milligrams of caffeine or a gelatine placebo. During exercise, ventilatory gas exchange parameters were measured breath by breath and at the end of each minute blood samples were drawn and lactate concentration determined. Ventilatory threshold (VT) and lactate threshold (LT) were defined as in the previously discussed literature. Comparisons of VT and LT were made between the caffeine and placebo trials.

No significant differences in maximal exercise values were shown between caffeine and placebo trials. When comparing the VO2 values for VT and LT during the placebo trials, no significant differences were found. However, during the caffeine trials, VT occurred at a significantly higher VO2 than did LT. VO2 for LT between the placebo and caffeine trials did not significantly differ. This disassociation suggested perhaps humoral 
or neural mechanisms were responsible for exercise-induced hyperpnea and provided further evidence that VT and LT were not always causally linked.

\section{Summary}

In this chapter, research supporting two different views on the relationship between ventilatory and lactate thresholds was discussed. Early research proposed that noninvasive measures of ventilatory changes during progressive exercise accurately represented changes in blood pH resulting from the accumulation of blood lactate (Caizzo et al., 1982; Koyal et al., 1976; Powers et al., 1984; Wasserman et al., 1973). In these studies, strong correlations were found between ventilatory breakaways, the accumulation of blood lactate, and metabolic acidosis. Additionally, other research showed that a ventilatory frequency breakaway was highly correlated with ventilatory threshold (James et al., 1989). This suggested that ventilatory frequency breakaway may be a good indicator of lactate threshold as well as ventilatory threshold. However, equally compelling and more recent research has shown that ventilatory and lactate thresholds were coincidentally related and easily disassociated. Subjects that did not produce lactate during progressive exercise demonstrated ventilatory breakaways similar to normal lactate producing subjects (Hagberg et al., 1982). Different training regimens resulted in different rates and magnitudes of change in ventilatory and lactate thresholds (Gaesser \& Poole, 1986; Poole \& Gaesser, 1985). Additionally, when subjects ingested caffeine prior to testing, ventilatory and lactate thresholds completely disassociated (Berry et al., 1991). Therefore, substantial disagreement continues to exist as to the relationship, if any, between the ventilatory and lactate thresholds. 


\section{Chapter III}

\section{Methods}

\section{Introduction}

This chapter provides information on the subjects, the study design and procedures, and the statistical analysis used for interpretation of the data.

\section{Subjects}

Twelve healthy, endurance trained male subjects aged 24 to 36 years were tested. Subjects performed aerobic exercise regularly according to the guidelines of the American College of Sports Medicine for developing and maintaining cardio-respiratory fitness. This included exercising at least 3-5 days per week for 20-60 minutes per session at $60-90 \%$ of age-predicted maximal heart rate (ACSM, 1990).

All subjects signed an informed consent form (Appendix A) and completed a health/medical history questionnaire (Appendix B) approved by the Human Subjects Institutional Review Board of San Jose State University.

Instrumentation

Maximal exercise tests were performed using a Monark cycle ergometer (model 818 ) with pedal straps. Minute by minute expired gas analysis was collected via a Wilmore-Costill Spinner Valve system. Expired gas samples were analyzed for fraction of $\mathrm{O} 2$ (FeO2) by an Applied Electronics S-3A O2 analyzer and for fraction of $\mathrm{CO} 2$ (FeCO2) by a Beckman Medical Gas CO2 Analyzer LB-2. Both gas analyzers were interfaced with an Apple II desktop computer that recorded data and calculated VE, VO2, VCO2, VE/VO2, VE/VCO2, and R for each minute of the test. A Yellow Springs Instruments (model 23L) Lactate Analyzer was used to determine blood lactate concentrations from blood samples drawn each minute during each test. A Narco Physiograph Recorder measured ventilatory 
frequency. Five lead ECG monitoring during testing was performed with a Nihon Kohden Cardiofax V Electrocardiograph (model ECG-8330A).

Procedures

Subjects were asked to refrain from exercise, food, alcohol, and caffeine two hours prior to testing. Upon reporting to the San Jose State University Exercise Physiology Lab, the subjects were advised of the testing procedures. They then completed the informed consent form and health/medical history questionnaire. Each subject was weighed prior to testing and skinfold measurements were taken to determine body composition. Subjects were given standardized instructions (Appendix C) directing them during the test to raise their right hand off the handlebars and signal to the evaluators if they noticed a sudden increase in their ventilation beyond that occurring with increased workloads.

For blood sampling, a sterile 22 gauge indwelling flexible catheter (BectonDickenson Angio-Set) was placed in a superficial vein on the dorsal aspect of the subject's left hand and then anchored to prevent movement. A registered nurse specializing in intensive care inserted the catheter using sterile procedures and performed all subsequent blood draws.

A rubber strap was wrapped around the subject's chest to hold two electrodes at the 5 th/6th intercostal space on the right and left sides of the trunk. These electrodes were attached to the Narco Physiograph Recorder that measured and recorded breathing frequency.

Before beginning the incremental cycle ergometer test, subjects were fitted with headgear that supported a Hans-Rudolph respiratory valve and mouthpiece. Subjects then performed an incremental maximal exercise test on the cycle ergometer. An initial warm-up workload of five minutes at zero Watts $(0 \mathrm{kgm} / \mathrm{min}$.) was followed by four minute stages with an increasing workload of 50 Watts $(300 \mathrm{kgm} / \mathrm{min}$.) each stage until maximum 
voluntary exhaustion. Four minute stages were selected to allow for metabolically produced lactate to completely diffuse into the bloodstream (Karlsson \& Jacobs, 1982;

Skinner \& McLellan, 1980). Subjects maintained a pedaling rate of $50 \mathrm{rpm}$. As a recovery period, subjects pedaled at zero Watts for 5 minutes or until heart rate decreased to under 120 beats per minute.

Testing was terminated if any one of the following conditions occurred: 1) when the subject met the previously described gas exchange and heart rate criteria for VO2 max, 2) when the subject could not maintain $50 \mathrm{rpm}$ for 30 or more seconds at the existing workload, 3) when the subject elected not to continue because of pain or fatigue, 4) if the subject demonstrated ECG abnormalities, 5) if the subject displayed abnormal signs of distress such as loss of coordination, pallor, or lightheadedness, 6) if the monitoring equipment failed. All tests were monitored by an American College of Sports Medicine certified Exercise Test Technologist trained in the administration and supervision of maximal exercise testing.

\section{Measurements}

Body Composition. Skinfold measurements were taken with Lange calipers (10 $\mathrm{g} / \mathrm{mm} 2$, constant pressure) on the right side of the body at the subscapular, triceps, midaxillary, and abdominal sites according to techniques described by Behnke and Wilmore (1974). Body density was determined using the equations described by Forsyth and Sinning (1973). Percent body fat was then calculated using the equations of Brozek et al. (1963). These procedures have been cross-validated for aerobically trained young adult males (Cisar, Housh, Johnson, Thorland, \& Hughes, 1989).

Gas Analysis. Gas analyzers were calibrated before each test with a known gas sample and then checked periodically during each test. Inhaled air passed through a Parkinson-Cowan CD-4 Dry Test Meter and then into the respiratory valve. The volume of 
inspired air was measured by a potentiometer interfaced with the Apple computer. Expired air passed out of the Hans-Rudolph valve and into a mixing chamber for analysis. Expired gas analysis measured the fraction of expired oxygen $(\mathrm{FeO} 2)$ and fraction of expired carbon dioxide $(\mathrm{FeCO} 2)$. From these measurements the computer calculated expired ventilation rate (VE BTPS in L/min.), oxygen uptake rate (VO2 in L/min. and $\mathrm{ml} / \mathrm{kg} / \mathrm{min}$.), carbon dioxide production rate (VCO2 in $\mathrm{L}$ min.), ventilatory equivalent for oxygen (VE/VO2), ventilatory equivalent for carbon dioxide ( $\mathrm{VE} / \mathrm{VCO} 2$ ), and the respiratory exchange ratio (R; VCO2/VO2). VE: VO2 was plotted for each minute of the test. Maximum oxygen uptake rate ( $\mathrm{VO} 2 \mathrm{max}$ ) and ventilatory threshold were determined by the criteria defined previously.

Ventilatory Frequency. A Narco Physiograph Recorder recorded breathing frequency by measuring pneumatic impedence via the two chest electrodes described above.

Lactate Analysis. At rest, during unloaded cycling, and at the end of each minute of exercise, the nurse drew a one $\mathrm{cc}$ (one $\mathrm{ml}$ ) blood sample from the venous catheter into a plastic three cc sterile heparinized syringe. Samples were stored in an ice bath for subsequent analysis by the lactate analyzer. Immediately following each test, the blood samples were injected into the lactate analyzer with a 25 microliter Yellow Springs Instruments Syringepet. The lactate concentrations for each minute sample were measured and recorded. The lactate analyzer was calibrated before each analysis with a sample solution of known lactate concentration as described in the procedures manual (Yellow Springs Instrument Co., 1988). The lactate threshold was determined by the criteria defined previously.

Perceived Ventilatory Threshold. The subjects signaled to the evaluators, as explained to them by the standardized intructions (Appendix C) given prior to the test, 
when they noticed a sudden increase in their ventilation rate and/or volume during the exercise test. The time and workload at which this occured was recorded and the corresponding physiological variables determined.

Heart Rate. Heart rate by five lead ECG was monitored continuously during the exercise test and recovery periods to measure heart rate (HR in $\mathrm{b} / \mathrm{min}$.) as well as detect unexpected cardiac dysrhythmias or electrical abnormalities.

\section{Statistical Analysis}

Descriptive statistics (means and standard deviations) for subject characteristics and the physiological variables measured at perceived ventilatory threshold (PVT), ventilatory threshold (VT), and lactate threshold (LT) were calculated. Repeated measures one-way analyses of variance (ANOVA) and Tukey post hoc tests were used to determine the mean differences between the physiological variables at PVT, VT, and LT. Pearson productmoment correlations were used to describe the relationships between physiological variables at PVT, VT, and LT. Statistical significance was set at alpha less than .05 . Statistical analyses were performed with the Statistical Package for the Social Sciences (SPSS). 


\section{Chapter IV}

Results

Introduction

This chapter contains the data anaysis, summary and discussion of results, conclusions, weaknesses of the study, and recommendations for future research. Analysis of Data

Descriptive Characteristics. Table 1 summarizes the descriptive characteristics of the twelve subjects tested. The average age of the subjects was $29.67 \pm 4.12$ years. Subjects' body weight and height averaged $79.64 \pm 10.23 \mathrm{~kg}$ and $178.42 \pm 4.26 \mathrm{~cm}$, respectively. Mean percent body fat was $15.45 \pm 6.49 \%$. Subjects had a mean VO2 max of $49.36 \pm 11.04 \mathrm{ml} / \mathrm{kg} / \mathrm{min}$.

Correlations. Tables 2 through 8 summarize the relationships found for physiological variables at PVT, VT, and LT. Strong correlations $(\underline{r} \geq .80 ; \underline{p}<.01)$ were found between VO2 at VT and LT ( $r=.92)$, time at PVT and VT $(r=.91)$, VO2 at PVT and LT $(\underline{r}=.88)$, VO2 at PVT and VT $(\underline{r}=.85)$, TV at VT and LT $(\underline{r}=.85), \mathrm{VF}$ at PVT and VT $(\underline{r}=.81)$, and TV at PVT and LT $(\underline{r}=.80)$. Moderate correlations $(.40<\underline{r}<.80 ; \underline{p}<.01)$ were found between VF and VE at PVT ( $r=79)$, time at PVT and LT ( $r=.76)$, VE at PVT and VT ( $(\underline{r}=.76)$, VF at PVT and LT $(\underline{I}=.73)$, and TV at PVT and VT $(\underline{I}=.73)$. Moderate correlations $(\underline{p}<.05)$ existed between VF at VT and $\mathrm{LT}(\underline{\mathrm{r}}=.68), \mathrm{VF}$ and $\mathrm{VE}$ at VT $(\underline{r}=.65)$, VE at PVT and LT ( $r=.65)$, and time at VT and LT $(r=.61)$.

Repeated Measures ANOVA. Table 9 summarizes the results of the one-way repeated measures ANOVA and Tukey post hoc analyses performed on the physiological variables measured at PVT, VT, and LT. Significant differences $(p<.05)$ between means for only PVT and VT were found in the following comparisons: 
Table 1

Descriptive Characteristics of the Subjects

$\underline{\mathrm{n}}=12$

Mean

SD

Age (years)

29.67

4.12

Body Weight (kg)

79.64

10.23

Height $(\mathrm{cm})$

178.42

4.26

Body Fat (\%)

15.45

6.49

$\mathrm{VO} 2 \max (\mathrm{ml} / \mathrm{kg} / \mathrm{min})$

$49.36 \quad 11.04$ 
Table 2

Zero Order Correlation Matrix for VO2 at PVT, VT, and LT $(n=12)$

VO2

PVT

VT

LT

PVT $\quad 1.00$

VT

$.85^{* *}$

1.00

LT

$.88^{* *}$

$.92 * *$

1.00

${ }^{*} \mathrm{p}<.05 .{ }^{* *} \mathrm{p}<.01$

Table 3

Zero Order Correlation Matrix for VE at PVT, VT, and LT $(n=12)$

$\begin{array}{lccc}\text { VE } & \text { PVT } & \text { VT } & \text { LT } \\ \text { PVT } & 1.00 & & \\ \text { VT } & .76^{* *} & 1.00 & \\ \text { LT } & .65^{*} & .35 & 1.00 \\ & & & \\ { }^{*} \underline{\mathrm{p}}<.05 .{ }^{* *} \mathrm{p}<.01 & & \end{array}$


Table 4

Zero Order Correlation Matrix for VF at PVT, VT, and LT

$(\mathrm{n}=12)$

$\begin{array}{llll}\text { VF } & \text { PVT } & \text { VT } & \text { LT }\end{array}$

PVT $\quad 1.00$

VT $\quad .81^{* *} \quad 1.00$

$\begin{array}{llll}\text { LT } & .73^{* *} & .68^{*} & 1.00\end{array}$

${ }^{*} \underline{p}<.05 .{ }^{* *} \underline{p}<.01$

Table 5

Zero Order Correlation Matrix for TV at PVT, VT, and LT

$(n=12)$

$\begin{array}{llll}\text { TV } & \text { PVT } & \text { VT } & \text { LT }\end{array}$

$\begin{array}{llll}\text { PVT } & 1.00 & & \\ \text { VT } & .73 * * & 1.00 & \\ \text { LT } & .80^{* *} & .85^{* *} & 1.00\end{array}$

${ }^{*} \underline{p}<.05 . *^{*} \underline{p}<.01$. 
Table 6

Zero Order Correlation Matrix for Time at PVT, VT, and LT

$(n=12)$

TIME

PVT

VT

LT

PVT $\quad 1.00$

VT

1.00

LT

${ }^{*} \mathrm{p}<.05 .{ }^{* *} \mathrm{p}<.01$

Table 7

Correlation Matrix for VF and VEat PVT, VT, and LT $(\mathrm{n}=12)$

PVTVE VTVE LTVE

$\begin{array}{lccc}\text { PVTVF } & .79 * * & .64^{*} & .59 * \\ \text { VTVF } & .46 & .65^{*} & .19 \\ \text { LTVF } & .22 & .14 & .46\end{array}$

${ }^{*} \mathrm{p}<.05 . \quad{ }^{* *} \mathrm{p}<.01$ 
Table 8

Correlation Matrix for TV and VE at PVT, VT, and LT $(n=12)$

PVTVE VTVE LTVE

$\begin{array}{llll}\text { PVTTV } & .68^{*} & .44 & .33 \\ \text { VTTV } & .26 & .24 & .19 \\ \text { LTTV } & .40 & .18 & .49\end{array}$

${ }^{*} \mathfrak{p}<.05 . \quad{ }^{* *} \mathfrak{p}<.01$. 
Table 9

Results of Repeated Measures ANOVA and Tukey post-hoc analyses for Physiological Variables at PVT, VT, and LT

\begin{tabular}{|c|c|c|c|c|c|c|}
\hline Variable & PVT & VT & LT & $F$ & p & $\mathrm{dT}^{\mathrm{b}}$ \\
\hline $\begin{array}{l}\mathrm{VO}^{\mathrm{a}} \\
(\mathrm{ml} / \mathrm{kg} / \mathrm{min})\end{array}$ & $34.79 \pm 3.79$ & $39.69 \pm 3.76$ & $39.03 \pm 2.82$ & 4.62 & .021 & 4.40 \\
\hline $\begin{array}{l}\mathrm{HR}^{\mathrm{a}} \\
(\mathrm{b} / \mathrm{min})\end{array}$ & $144.08 \pm 5.63$ & $157.00 \pm 4.05$ & $155.25 \pm 3.42$ & 4.22 & .028 & 12.13 \\
\hline $\begin{array}{l}V^{a} \\
\text { (L/min) }\end{array}$ & $62.42 \pm 5.96$ & $75.58 \pm 3.97$ & $72.13 \pm 3.38$ & 5.18 & .014 & 10.67 \\
\hline $\begin{array}{l}\mathrm{VF} \\
\text { (breaths/min) }\end{array}$ & $24.75 \pm 1.76$ & $27.67 \pm 1.71$ & $26.92 \pm 1.41$ & 3.23 & .059 & N/A \\
\hline $\begin{array}{l}\text { TVa } \\
\text { (L/breath) }\end{array}$ & $2.51 \pm .15$ & $2.79 \pm .14$ & $2.72 \pm .14$ & 5.01 & .016 & .23 \\
\hline $\begin{array}{l}\mathrm{LA} \\
(\mathrm{mM} / \mathrm{L})\end{array}$ & $2.26 \pm .28$ & $2.63 \pm .33$ & $2.34 \pm .19$ & 1.36 & .227 & $\mathrm{~N} / \mathrm{A}$ \\
\hline $\mathrm{RPE}^{\mathrm{a}}$ & $14.33 \pm .74$ & $15.50 \pm .47$ & $15.25 \pm .37$ & 3.70 & .041 & 1.13 \\
\hline$\%$ VO2 $\max ^{\mathrm{a}}$ & $69.25 \pm 4.49$ & $79.96 \pm 2.26$ & $78.82 \pm 1.45$ & 4.52 & .023 & 9.84 \\
\hline$\% H R \max ^{a}$ & $78.79 \pm 3.08$ & $85.73 \pm 1.73$ & $84.81 \pm 1.49$ & 4.05 & .032 & 6.66 \\
\hline
\end{tabular}

Note. PVT, VT, $\mathrm{LT}$ values are $\underline{\mathrm{M}} \pm \mathrm{SEM}$.

aOne way analysis of variance significant at $\underline{p}<.05$ and Tukey post hoc difference between PVT and VT means only.

$\mathrm{b}_{\mathrm{dT}}$ is the minimum pairwise difference between means that must be exeeded to be significant with the Tukey test. 
VO2 $(34.79 \pm 3.79$ vs. $39.69 \pm 3.76, \mathrm{p}=.021), \mathrm{HR}(144.08 \pm 5.63$ vs. $157 \pm 4.05$, $\mathrm{p}=.028), \operatorname{VE}(62.42 \pm 5.96$ vs. $75.58 \pm 3.97, \mathrm{p}=.014), \mathrm{TV}(2.51 \pm .15$ vs. $2.79 \pm .14$, $\mathrm{p}=.016), \operatorname{RPE}(14.33 \pm .74$ vs. $15.50 \pm .47, \mathrm{p}=.041), \% \mathrm{VO} 2 \max (69.25 \pm 4.49$ vs. $79.96 \pm 2.26, \mathrm{p}=.023)$, and $\% \mathrm{HR} \max (78.79 \pm 3.08$ vs. $85.73 \pm 1.73, \mathrm{p}=.032)$. No other significant differences were detected between the means of other physiological variables measured for PVT and VT. Also, no significant differences were found between the means on any of the physiological variables measured for either PVT and LT or VT and LT.

\section{Summary of Findings}

The physiological values at PVT and VT, PVT and LT, and VT and LT for VO2 had the strongest correlations. For VE, PVT and VT, and PVT and LT were each moderately correlated while VT and LT were weakly related. The VF at PVT and VT were strongly related while VF at PVT and VT and LT and VT were only moderately correlated. TV measures had strong positive relationships between PVT and LT and VT and LT. A moderate correlation was found between TV at PVT and TV at VT. The time for PVT and VT were strongly related, but the times for VT and LT and for PVT and LT were only moderately related.

An evaluation of the relationships between the two components of ventilation (TV and VF) and expired ventilation rate (VE) indicated a relatively strong relationship existed between VF and VE at PVT. Of the remaining relationships between VF and VE, only VF and VE at VT were moderately related. TV and VE were moderately correlated only at PVT.

No significant differences were found between the means of VT and LT on any of the physiological variables measured. On all of the variables measured except for VF and LA, significant differences were found between the means of PVT and VT. Additionally, 
on these statistically significant measures. PVT occurred at a consistently lower or earlier value than did VT. No statistically significant differences were observed between the means of PVT and LT on any of the physiological variables measured.

However, the Bonferroni correction for multiple comparisons was not applied in this study. This more stringent test of statistical significance would have reduced the alpha level for each comparison according to the formula .05 / number of comparisons. In this study alpha would have been reduced to $\mathrm{p}<.006$. Under these conditions, none of the comparisons between PVT, VT, and LT would have been statistically significant.

\section{Discussion of Findings}

The correlation in this study between VT and LT for VO2 ( $r=.92)$ was similar to the findings of Caiozzo et al. (1982) who found a strong correlation ( $r=.93$ ) between VT and LT. Powers et al. (1984) used a group of highly trained males similar to the subjects in this study. In their study VT and LT occurred at exactly the same VO2 in six of thirteen subjects. Similarly, in this study VT and LT occurred at exactly the same time and consequently the same VO2 in four of twelve subjects. However, these results must be interpreted carefully as breath by breath gas analysis was performed in the Powers et al. study, whereas less precise minute by minute analysis was performed in this study. Nonetheless, the strong relationships between the VO2 at PVT and VT $(r=.85)$ and the VO2 at PVT and LT ( $(\underline{=}=88)$ suggest that the perception of ventilatory threshold may be a good indicator of both the ventilatory and lactate thresholds. Interestingly, these findings are in direct contrast with literature that proposed the coincidental finding of relationships between VT and LT and described them as two unrelated events (Berry et al., 1991; Hagberg et al., 1982; Gaesser \& Poole, 1986; Poole \& Gaesser, 1985).

The relationship between time at PVT and time at VT was the second highest correlation ( $r=.91)$ in the data analysis. This indicated a close link between the time at 
which ventilatory changes actually occur and when they are perceived to occur. Additionally, the time of PVT was a good indicator of the time at which LT occurred $(\underline{r}=.76)$. Again, some care should be taken in the interpretation of these particular results as both ventilatory and lactate thresholds were recorded on a whole minute basis and PVT was extrapolated to a whole minute value from a more precise minute and seconds report during the test.

When comparing the components of expired ventilation rate, ventilatory frequency and tidal volume, the results indicated a much stronger correlation between VF and VE at VT than between TV and VE at VT. The only statistically significant correlation, although moderate ( $(\underline{r}=.68)$, for TV occurred between the TV and VE at PVT. This was not as strong as the relationship between the VF and VE at PVT ( $r=79)$. These results suggest that breathing frequency (VF) may be the more important ventilatory cue for perceiving ventilatory threshold than breathing depth (TV), although both appear to be somewhat important in perceiving ventilatory threshold. This supports previous research which found that at higher exercise intensities, increases in ventilatory frequency were the primary feature of the respiratory compensation to metabolic acidosis (Wasserman, 1978). Additionally, this supports previous literature which stated that ventilatory frequency was a primary cue for the perception of physical effort (Mihevic, 1981).

Results of the repeated measures ANOVA and Tukey post hoc analyses revealed significant differences between PVT and VT in all of the comparisons except for lactate concentration and ventilatory frequency. In all of the physiological variables measured, no significant differences were found between either PVT and LT or VT and LT. Interestingly, these results indicated that although PVT occurred earlier and at lower physiological values than VT, PVT did not occur significantly earlier or at lower physiological values than LT. Hence, PVT appears to be a better indicator of LT than of 
VT. The ability to detect LT through PVT provides an important tool in monitoring exercise intensity at or above the anaerobic threshold. The ability to accurately reproduce LT level exercise may be more important than detecting VT for the competitive or serious recreational athlete. LT has been considered the more important measure associated with high level endurance performance and aerobic capacity than has VT (Hagberg, 1984; McArdle et al., 1991).

Because no significant differences were found between the ventilatory frequency at PVT, VT, or LT, the perception of breathing rate alone may be the most accurate indicator of both threshold measures. These results support other findings in this study that indicated VF to have a stronger relationship with VE than TV at either VT or LT. These findings also support James et al. (1989) who reported the VF threshold was a strong indicator of the VE threshold. Since lactate was also measured in the present study, the results strengthen the possibility of using VF as an indicator of LT.

Close examination of the raw data shows that PVT occurred at a consistently lower and earlier value than either VT or LT in all of the physiological variables measured. For the subjects in this study, VT and LT occurred at nearly a 10\% higher relative exercise intensity when expressed as percentage of VO2 max and HR max. Additionally, VT and LT occurred at an HR over ten beats per minute higher than the HR at PVT. This suggests that for an individual to reach actual anaerobic threshold training levels, as determined by either lactate or ventilatory breakaways, the individual should continue exercise to a point slightly higher than PVT. However, the mean PVT values for both \%HR max $(78.79 \pm$ $3.08 \%)$ and $\%$ VO2 $\max (69.25 \pm 4.49 \%)$ occured well within the exercise intensity range for general aerobic conditioning (60-90\% of HR max and $50-85 \%$ of VO2 max) as recommended by the American College of Sports Medicine (1990). This suggests that although PVT was not a precise indicator of ventilatory or lactate thresholds, PVT was a 
good indicator of appropriate exercise intensity for the development of cardiorespiratory fitness. Therefore, PVT may be used as an accurate exercise intensity monitoring tool for general aerobic training.

\section{Conclusions}

Within the limits of this study the following conclusions were made:

1. Ventilatory frequency was the most accurate ventilatory cue for determining both lactate and ventilatory thresholds.

2. PVT was a better indicator of LT than of VT on most of the physiological variables measured.

3. PVT was consistently detected earlier than both VT and LT.

4. VT and LT were not statistically or practically different, suggesting that ventilatory threshold was the respiratory response to the accumulation of blood lactate and metabolic acidosis.

\section{Practical Applications}

The following practical applications can be made from the conclusions drawn from this study:

1. For exercising subjects to actually achieve LT or VT levels of exercise, they must exercise at a slightly higher intensity than PVT.

2. PVT can be used as an accurate indicator for appropriate exercise intensity during general cardiorespiratory conditioning.

\section{Weaknesses of the Study}

The primary weakness of this study was the small sample size due to equipment failure mid-way through data collection. Because of the possibility of a type II error under these conditions, the non-significant findings between PVT and LT may not provide strong enough evidence to show that PVT and LT occur together, at the same time, or at the same 
physiological values.

Additionally, all gas analysis and lactate measurements were made on a whole minute basis while the report of PVT was extrapolated to a whole minute value from a more precise measure of both minutes and seconds. Whether a PVT detected especially early, in the middle, or especially late in a minute would have values closer to the minute following or the minute within which it was recorded remains unclear.

\section{Recommendations for Future Research}

Within the limits of this study, the following recommendations for future research are made:

1. Future research should use equipment capable of breath by breath analysis to improve the continuity of data collected so that more accurate comparisons can be made.

2. Future research should examine whether or not training subjects to more closely monitor their ventilation frequency can enhance their ability to detect the ventilatory threshold.

3. Future research should examine whether the results obtained in this study apply to other types of aerobic activity (eg., running).

4. Future research should examine whether subjects of varied aerobic capacities and/or varied years of continuous aerobic training differ in their abilities to detect ventilatory threshold.

5. Future research should examine the responses and abilities of female subjects to detect the ventilatory threshold.

6. Future research should examine the accuracy with which untrained subjects can detect the ventilatory threshold and whether or not this ability improves with improvements in aerobic fitness and with regular aerobic training. 
References

American College of Sports Medicine. (1991). Guidelines for exercise testing and prescription (4th ed.). Philadelphia: Lea and Febiger.

American College of Sports Medicine. (1990). The recommended quantity and quality of exercise for maintaining cardiorespiratory and muscular fitness in healthy adults. Medicine and Science in Sports and Exercise, 22 (2), 265-274.

Behnke, A.R., \& Wilmore, J.H. (1974). In Evaluation and Regulation of Body Build and Composition (pp. 39-50). Englewood Cliffs, New Jersey: Prentice Hall

Berry, M.J., Stoneman, J.V., Weyrich, A.S., \& Burney, B. (1991). Disassociation of the ventilatory and lactate thresholds following caffeine ingestion. Medicine and Science in Sports and Exercise, 23 (4), 463-469.

Brooks, G.A. (1985). Anaerobic threshold: Review of the concept and directions for future research. Medicine and Science in Sports and Exercise, 17 (1) 22-31.

Brooks, G.A., \& Fahey, T.D. (1984). Metabolic response to exercise. Exercise Physiology: Human Bioenergetics and Its Applications (pp. 189-219). New York: John Wiley \& Sons.

Brozek, J., Grande, F., Anderson, J.T., \& Keys, A. (1963). Densitometric analysis of body composition: review of some quantitative assumptions. Annals of the New York Academy of Science, 110, 113-140.

Caiozzo, V.J., Davis, J.A., Ellis, J.F., Azus, J.L., Vandagriff, R., Prietto, C.A., \& McMaster, W.C. (1982). A comparison of gas exchange indices used to detect anaerobic threshold. Journa! of Applied Physiology, 53 (5), 1184-1189. 
Cisar, C.J., Housh, T.J., Johnson, G.O., Thorland, W.G., \& Hughes, R.A. (1989). Validity of anthropometric equations for determination of changes in body composition in adult males during training. Journal of Sports Medicine and Physical Fitness, 29 (2), 141-148.

Davis, J.A. (1985). Anaerobic threshold: Review of the concept and directions for future research. Medicine and Science in Sports and Exercise, 17 (1), 6-18.

Davis, J.A., Caiozzo, V.J., Lamarra, N., Ellis, J.F., Vandagriff, R., Prietto, C.A., \& McMaster, W.C. (1983). Does the gas exchange anaerobic threshold occur at a fixed blood lactate concentration of 2 or $4 \mathrm{mM}$ ? International Journal of Sports Medicine, 4, 89-93.

Gaesser, G.A., \& Poole, D.C. (1986). Lactate and ventilatory thresholds: Disparity in time course of adaptations to training. Journal of Applied Physiology 61, 9991004.

Hagberg, J.M. (1984). Physiological implications of the lactate threshold. International Journal of Sports Medicine, 5 , 106-109.

Hagberg, J.M., Coyle, E.F., Carroll, J.E., Miller, J.M., Martin, W.H., \& Brooke, M.H. (1982). Exercise hyperventilation in patients with McArdle's disease. Journal of Applied Physiology: Respiratory Environmental Exercise Physiology, 52 (4), 991 994.

James, N.W., Adams, G.M., \& Wilson, A.F. (1989). Determination of anaerobic threshold by ventilatory frequency. International Journal of SportsMedicine, 10 (3), 192-196.

Karlsson, J., \& Jacobs, I. (1982). Onset of blood lactate accumulation during muscular exercise as a threshold concept: Theoretical considerations. International Journal of Sports Medicine, 3 , 190-201. 
Koyal, S.N., Whipp, B.J., Huntsman, D., Bray, G.A., \& Wasserman, K. (1976). Ventilatory responses to the metabolic acidosis of treadmill and cycle ergometry. Journal of Applied Physiology, 40 (6), 864-867.

McArdle, W.D., Katch, F., \& Katch, V. (1991). Pulmonary ventilation during exercise. Exercise Physiology: Energy, Nutrition, and Human Performance (pp. 278291). Philadelphia: Lea \& Febiger.

Mihevic, P.M. (1981). Sensory cues for perceived exertion: A review. Medicine and Science in Sports and Exercise, 13 (3), 150-163.

Poole, D.C., \& Gaesser, G.A. (1985). Response of ventilatory and lactate thresholds to continuous and interval training. Journal of Applied Physiology, $\underline{58}$ (4), 11151121.

Powers, S.K., Dodd, S., \& Garner, R. (1984). Precision of ventilatory and gas exchange alterations as a predictor of the anaerobic threshold. European Journal of Applied Physiology, 52, 173-177.

Skinner, J. S., \& McLellan, T. H. (1980). The transition from aerobic to anaerobic metabolism. Research Quarterly for Exercise and Sport, 51 (1), 292-296.

Wasserman, K. (1978). Breathing during exercise. New England Journal of Medicine, $\underline{298}, 780-785$.

Wasserman, K., Whipp, B.J., Koyal, S.N., \& Beaver, W.L. (1973). Anaerobic threshold and respiratory gas exchange during exercise. Journal of Applied Physiology, 35, 236-243.

Wasserman, K., Whipp, B.J., Koyal, S.N., \& Cleary, M.G. (1975). Effect of carotid body resection on ventilatory and acid-base control during exercise. $\underline{\text { Journal of }}$ Applied Physiology, 39, 354-358. 
Whipp, B.J. (1983). Ventilatory control during exercise in humans. Annual Reviews of Physiology, 45, 393-413.

Whipp, B.J., \& Davis, J.A. (1979). Peripheral chemoreceptors and exercise hyperpnea. Medicine and Science in Sports and Exercise, 11 (2), 204-212.

Yellow Springs Instrument Company, Inc. (1988). YSI Model 23L Lactate Analyzer

Instruction Manual, Yellow Springs, OH: Author. 


\section{APPENDIX A}

\section{Agreement to Participate in Research}

San Jose State University

Department of Human Performance

Responsible Investigator(s):

Title of Protocol: THE PERCEPTION OF VENTILATORY THRESHOLD AND ITS

\section{ACCURACY FOR INDICATING ANAEROBIC THRESHOLD}

1. I have been asked to participate in a research study investigating the ability of an exercising subject to accurately perceive ventilatory threshold and whether the ventilatory threshold is an accurate indicator of lactate threshold.

2. I will be invited to the San Jose State Department of Human Performance for a single graded maximal cycle ergometer exercise test. This test will determine my maximal aerobic capacity / maximal oxygen consumption. Before beginning the test, all procedures will be fully explained. Two electrodes to measure my breathing frequency will be attached to my chest. A small intravenous catheter will be inserted into the back of my left hand so that blood samples may be drawn during the test for subsequent analysis. The actual exercise test will require me to pedal a stationary bicycle at progressively increasing workloads until I can no longer move the pedals at a predetermined rate. At this time, the resistance on the bicycle will be reduced so that I may complete a cool down period of no less than five minutes. During the entire test expired air will be collected through a mouthpiece attached to rubber tubing. In addition, my heart rate will be monitored continuously throughout the test on an electrocardiograph via electrodes placed on my chest. Small (one cc) blood samples will be drawn from the flexible catheter at minute intervals immediately before, during, and after the test. A total of 10-30 cc's of blood will be drawn over the entire test.

3. I may experience some minor discomfort as the catheter is inserted into the back of my hand. During the test, I may experience some discomfort and dryness in the mouth, throat, and chest due to breathing through the mouthpiece. I may also experience discomforts commonly associated with exercise; sweating, increased heart rate, increased breathing rate, increased body temperature, and fatigue. Changes that may be anticipated at or near maximal exercise include abnormal blood pressure, fainting, dizziness, muscle fatigue or cramps; nausea; and abnormalities in heart beat (some of these could result in death). Individuals will be excluded from this investigation when abnormalities are detected in electrocardiograph recordings or medical history indicates that testing is contraindicated. During exercise I may experience heartbeat abnormalities; if these are detected the test will be stopped immediately. I may be extremely fatigued, lightheaded, and slightly nauseous for a short time following the exercise test.

4. I will benefit from this study by receiving an accurate and complete assessment of my cardiorespiratory fitness. This information may be used as the basis of an aerobic training program.

Initial 
5. The data generated may be used for medical and/or scientific purposes, including publication and presentation at professional meetings. My name will not be revealed unless my expressed written consent is granted prior to publication or presentation of the data.

6. Any questions regarding this research may be addressed to Howard Lieberman at (408) 257-9590. Complaints about the research may be presented to Craig Cisar, Ph.D. (thesis advisor) at (408) 924-3018. Questions or complaints about the research, subjects' rights, or research related injury may be presented to Serena Sanford, Ph.D., Associate Vice President of Graduate Studies and Research, at (408) 924-2480.

7. I may refuse to participate in this study or in any part of this study and I may withdraw at any time without prejudice to my relations with San Jose State University.

8. My consent to participate in this research is given voluntarily without being coerced.

9. I have received a signed, dated copy of this consent form for my records.

*The signature of a subject on this document indicates agreement to participate in this study.

**The signature of a researcher on this document indicates agreement to include the above named subject in the research and attestation that the subject has been fully informed of his or her rights.

Subject's Signature and Date

Investigator's Signature and Date 


\section{APPENDIX B}

\section{Health/Medical History Questionnaire}

\section{PERSONAL INFORMATION}

Date

Name

Age Birthdate

Address

Home Phone

Occupation

Work Phone

Personal Physician

Physician Phone

\section{MEDICAL IISTORY}

Check if you previously or currently have any of the following conditions:

$\begin{array}{llll}\text { heart disease } & () & \text { cough on exertion } & () \\ \text { heart attack } & () & \text { leg/ankle swelling } & 0 \\ \text { high blood pressure } & () & \text { kidney problems } & () \\ \text { high blood cholesterol } & () & \text { rheumatic fever } & () \\ \text { high blood triglycerides } & () & \text { varicose veins } & 0 \\ \text { diabetes } & () & \text { surgeries } & () \\ \text { chest pain } & 0 & \text { list: } & \\ \text { joint/muscle injury } & 0 & & 0 \\ \text { heart murmur } & 0 & \text { back problems } & () \\ \text { heart palpitations } & () & \text { epilepsy } & () \\ \text { arterial disease } & () & \text { arthritis } & () \\ \text { lung disease } & () & \text { anemia } & () \\ \text { shortness of breath } & () & \text { hernia } & 0 \\ \text { coughing of blood } & () & \text { medications } & \\ \text { dizziness } & () & \text { list: } & \\ \text { other(s) } & () & & \end{array}$

Initial 
Have you ever had a maximal exercise test? yes() no()

If yes, what was the result?

\section{RISK FACTORS}

1. Smoking: yes no

$\begin{array}{llll}\text { cigarettes } & () & 0 & \text { \# per day__ \# of years__ } \\ \text { cigars } & 0 & 0 & \text { \# per day__ \# of years___ } \\ \text { pipe } & 0 & \text { O } & \text { \# per day__ of years__ }\end{array}$

2. Weight: now 1 yr ago 3 yrs ago

\section{Exercise Habits}

type

years of consistent training

frequency: days per week

duration: minutes per session

intensity: long steady distance ()

interval training $\quad()$

sprint training ()

\section{Occupation}

Physical Demand Level of Occupation

sedentary

light work

()

medium work

()

heavy work

()

\section{Lifestyle}

physically active

sedentary

()

()

stressful

Initial 


\section{Family History}

Check if any blood relatives (parents, brothers, sisters, aunts, uncles, grandparents) have or had any

of the following:

$\begin{array}{ll}\text { heart disease } & () \\ \text { heart attack prior to age 50 } & () \\ \text { stroke prior to age } 50 & () \\ \text { elevated blood cholesterol } & () \\ \text { elevated blood triglycerides } & () \\ \text { high blood pressure } & () \\ \text { diabetes } & ()\end{array}$

Signed

Date 


\begin{abstract}
APPENDIX C
Pretest Instructions for Signaling the Perception of Ventilatory Threshold

During the exercise test, you will notice a gradual increase in your breathing that corresponds to the gradual increase in work you will be performing. However, at some point during this progression you may experience a sudden increase in your breathing rate or volume; much the same as you might experience if riding a bicycle from flat to hilly terrain or if changing a running pace from a jog to a sprint. Please signal to the evaluators if you notice this occurence by raising your right hand off the handlebars of the bicycle and pointing your right index finger towards the ceiling. You will be acknowledged by the evaluators and may then return your hand to the handlebars for the remainder of the test.
\end{abstract}

Do you understand these instructions?

Do you have any questions regarding these instructions?

Signed

Date 